De ficciones, tecnologías, controles y terrores: Psyco-Pass entre el poder, producción, normalización y la resistencia

Martín Ariel Gendler

Question, Vol. 1, N. 57, e031, enero-marzo 2018. ISSN 1669-6581

http://perio.unlp.edu.ar/ojs/index.php/question/article/view/4317

FPyCS | Universidad Nacional de La Plata

La Plata | Buenos Aires | Argentina

Recibido: 31-07-2017 Aceptado: 14-11-2017

Cita sugerida: Gendler, M. (2018). De ficciones, tecnologías, controles y terrores: Psyco-Pass entre el poder, producción, normalización y la resistencia. Question, 1(57), e031. doi: https://doi.org/10.24215/16696581e031

\title{
De ficciones, tecnologías, controles y terrores: Psyco-Pass entre el poder, producción, normalización y la resistencia
}

\author{
From fictions, technologies, controls and terrors: Psycho-Pass between \\ power, production, normalization and resistance
}

\author{
Martín Ariel Gendler \\ Instituto de Investigaciones Gino Germani; Facultad de Ciencias \\ Sociales; Universidad de Buenos Aires/ Consejo Nacional de \\ Investigaciones Científicas y Técnicas (Argentina) \\ martin.gendler@gmail.com
}

\section{Resumen}

El presente trabajo tiene como objetivo realizar un análisis minucioso del anime japonés PsycoPass el cual, ambientado en un futuro signado por una configuración social de gran avance y control tecnológico, presenta un marco narrativo sumamente interesante para el análisis sociológico.

En Psyco-Pass diversos dispositivos tecnológicos, prácticas, discursos y saberes se combinan para configurar un orden social en la que cada individuo tiene inscripto en su cuerpo un 
"coeficiente de criminalidad", que indica su estatus dentro de la gama de lo correcto, lo aceptable y lo imposible/eliminable. Asimismo, tanto este coeficiente como los servicios de seguridad e incluso las disposiciones más simples de la vida cotidiana son supervisados y orientados por el sistema Sybil, un sistema informático conformado (aparentemente) por algoritmos y patrones de conducta que clasifica y ordena la vida social en este Japón futurista. Siguiendo estos parámetros, la narración circula por varios parajes donde personajes principales, secundarios y antagonistas deben enfrentarse a diversas situaciones prácticas, morales y de poder actuando en conformidad, en duda o en total sublevación contra el orden imperante.

En síntesis, Psyco-Pass nos abre una ventana a una potencial configuración social no tan lejana a la actual que invita a su análisis y reflexión.

Palabras clave: control tecnológico; ciencia ficción; resistencia; configuración social.

Abstract

This work aims to make a detailed analysis of the Japanese anime Psyco-Pass which, set in a future marked by a social configuration of great advance and technological control, presents a very interesting narrative framework for sociological analysis.

In Psyco-Pass technological devices, practices, speeches and knowledge combine to form a social order where each individual has a 'crime coefficient' inscribed in his body which indicates his status within the range of the right, the acceptable and the impossible/removable. Likewise, both this coefficient as the security services and even the simpler provisions of daily life are supervised and guided by the Sybil system, a computer system made up (apparently) of algorithms and behavior patterns that classifies and orders social life in this futuristic Japan.

Following these parameters, the narrative circulates through several places where various main, secondary and antagonistic characters must face various practical, moral and power situations acting in conformity, in doubt or in total uprising against the prevailing order.

In short, Psyco-Pass opens a window to a potential social configuration not so far away from the current one that invites its analysis and reflection.

Keywords: technological control; science fiction; resistance; social configuration. 
"Las historias de ciencia ficción son

viajes extraordinarios a uno

de los infinitos futuros concebibles".

Isaac Asimov, Sobre la Ciencia Ficción

A la hora de investigar, el/la sociólogo/a enfrenta diversos desafíos como ver la factibilidad de su objeto de estudio, recortarlo en un espacio temporal determinado, seleccionar las herramientas metodológicas adecuadas para su abordaje y especialmente mantener una vigilancia epistemológica (Bourdieu, Chamboredon y Passeron, 2002) durante todo el proceso que le permita esgrimir su investigación como objetiva y válida.

En definitiva, de lo que se trata es de investigar lo social, la sociedad o, quizás enfocando un poco, las relaciones sociales surgidas, difundidas, mantenidas, potenciadas, etcétera, por virtud y obra de los diversos individuos, colectivos, instituciones, sistemas, mecanismos.

Es decir, el/la sociólogo/a debe enfocarse en estudiar las relaciones sociales y, por tanto, relaciones de poder (Marin, 1996; Elias, 1994) en sus diversos lineamientos, escondrijos, recursos, producciones, batallas, asociaciones, alianzas, reglamentos y normas, subjetividades e identidades.

Sin embargo, pareciera como si la sociología en líneas generales (1) se hubiera olvidado de trabajar con algunas de las producciones sociales más fructíferas en lo que respecta a potenciales configuraciones, relaciones y creaciones sociales: la ciencia ficción en sus diversas aristas. Así, las obras y los autores de este género parecieran solo ser retomados como recursos narrativos a la hora de describir o analizar situaciones sociales 'reales' análogas, pero no un análisis sociológico de las mismas. De esta forma tenemos leyes "asimoveanas", contextos "orwelleanos" o educaciones "huxleyeanas" entre muchos otros ejemplos.

El punto es que la sociología en general, pareciera no querer adentrarse en los relatos, situaciones, configuraciones y relaciones sociales que son elaboradas y desplegadas al interior de las producciones de ciencia ficción, las cuales, como expresa la frase que da inicio a esta sección, representan diversas problemáticas, anhelos, deseos, miedos y potenciales de futuro (deseables o no) posibles. La ciencia ficción revela mucho de la sociedad; configuraciones y relaciones donde es elaborada, amén del aporte individual de su autor.

En el presente trabajo nos proponemos realizar un análisis sociológico de una obra de ciencia ficción, del anime Psycho-Pass (2) dirigido por Naoyoshi Shiotani y Kiyotaka Suzuki, producido por el estudio Production I. G. y emitido a finales de 2012 primero en Japón y luego en diversos países del mundo.

Sumándolo a la lista antes mencionada, el anime ha incursionado fructíferamente en la ciencia ficción desde 1961 con el cortometraje 50000 años después de Shinichi Suzuki y Astro Boy en 
1963, pasando por otros animes más recientes como Cowboy Bebop, Neon Génesis Evangelion, Ghost in the Shell, Battle Royale, entre muchos otros.

El anime como formato le da a la ciencia ficción (entre muchas otras cosas) ciertos permisos y ventajas para expresar y mostrar situaciones que serían difíciles de ver en actores de carne y hueso y cierta dinámica que las restringidas viñetas del cómic y del manga restringen, ayudándonos de este modo a sumergirnos en un mundo extraño y familiar a la vez.

\section{Del saber-poder, la estadística, el control, el miedo y sus producciones}

"Lo que hace que el poder se sostenga, que sea aceptado, es sencillamente que no pesa como potencia que dice no, sino que cala de hecho, produce cosas, induce placer, forma saber, produce discursos; hay que considerarlo como una red productiva".

(Foucault, 1981: 137)

Siguiendo a Elias (1989) entendemos a las relaciones de poder como un elemento sustancial y central del análisis sociológico, usualmente descuidado, sin el cual nuestro análisis podría caer en un terreno de esterilidad. En este sentido, al hablar de poder en realidad nos referimos a relaciones de poder (Foucault, 1987; Elias, 1994); a una red dinámica y productiva de prácticas, acciones y relaciones sociales de interdependencia, conflicto y mutua influencia entre los sujetos, inserta en una configuración histórico-social determinada, que a la vez modifica.

Podemos ver en Foucault a uno de los más férreos opositores al abordaje del poder como objeto situado plausible de ser transferido o ejercido para la represión. En este sentido, el poder no es una cosa; es una estrategia que no se posee, sino que se ejerce (principalmente a través de múltiples redes, tecnologías y dispositivos conectados) de forma productiva: produce cuerpos, subjetividades/subjetivaciones, prácticas, disposiciones y campos para la acción; produce autoridades y disciplinas; produce saberes, deseos y verdades, produce normas y normalizaciones, en definitiva produce "efectos de poder":

Lo que define una relación de poder es un modo de acción que no actúa directa e inmediatamente sobre los otros, sino que actúa sobre su propia acción. Una acción sobre la acción, sobre acciones eventuales o concretas, futuras o presentes (Foucault 1984: 3). 
Por su parte, Norbert Elias centra la mirada sociológica en la explicación de las relaciones de poder, entendiéndolas como una cadena de interdependencias en constante tensión; como una balanza cambiante de equilibraciones, flujos y reflujos, grados, alianzas y enemistades inserta en una configuración histórico-social determinada (y sin embargo en producción constante) de relaciones sociales entre individuos, roles, grupos e instituciones; como relaciones polimorfas y multicausales; como coacciones y contra-coacciones; como relaciones dinámicas que se forman, se construyen, se ejercitan, se transforman y se destruyen. En palabras del propio autor:

(...) lo que llamamos "poder" es un aspecto de una relación, de cada una de las relaciones humanas (1994: 53-54); (...) Es como si miles de personas, primero, luego millones y finalmente más y más millones anduvieran por este mundo con los pies y las manos atados a los demás por ataduras invisibles. Nadie guía ese andar. Nadie queda fuera de él. Algunos quieren ir hacia allí, otros hacia allá. Caen unos sobre otros, y vencedores y vencidos siguen encadenados entre sí. Nadie puede dirigir los movimientos del todo (1990: 20-21).

Siguiendo a Juan Carlos Marín (1996), es a partir del enfrentamiento, de la ruptura, de la confrontación, de la guerra que se funda lo social, se crean (y destruyen) relaciones sociales de fuerza material y por tanto de poder y saber; se enuncian y crean diversas territorialidades sociales; se expropian y producen cuerpos, corporalidades y prácticas; se crean y se ponen en ejercicio armas materiales y morales; se establece la "paz" como un falso equilibrio del vencedor signado y (re)estructurado por este, enmascarando los combates y enfrentamientos que dieron lugar a esa situación de "paz".

\section{Estadística, seguridad y biopolítica}

A estas concepciones se agregan los procesos biopolíticos los cuales, siguiendo a Foucault (1996) y Sibilia (2005), fueron implementados en el siglo XIX gracias a la acumulación de conocimientos basados en la observación y el examen y en la capacidad de realizar un procesamiento centralizado de esa información (vía la estadística y la demografía).

Cabe destacar que, en estos mecanismos de seguridad nacientes en este proceso, se efectúa un procedimiento de normalización, es decir, de "corrimiento de la norma" donde las normas fijas características de los dispositivos disciplinares anatomopolíticos van mutando y adaptándose en torno a las curvas y parámetros estadísticos de normalidad y anormalidad del régimen de verdad, saber y poder, configurando lo que es deseable/correcto, lo posible y lo indeseable/incorrecto. 
En este sentido, los dispositivos de seguridad realizan otra interesante operación: “(...) la regulación de un medio no se trata tanto de fijar los límites y las fronteras ni tampoco de determinar las posiciones, sino de, esencialmente, permitir, garantizar y asegurar las circulaciones de personas, mercaderías, etc." (Foucault, 2006: 31). Este tipo de dispositivos se caracterizan por determinar estos espacios de seguridad, el cálculo estadístico y probabilístico, la normalización y la correlación entre técnicas de seguridad y población donde los sujetos no están encerrados, sino que circulan de forma constante y son monitoreados en este andar.

Así, el "sometimiento" se transfigura a gubernamentalidad, entendida como "técnicas y procedimientos destinados a dirigir las conductas de los hombres" (Foucault, 1980: 125) donde una serie de posibilidades para la acción correctas y posibles (y no otras) son desplegadas ante los sujetos de acuerdo a los parámetros normalizados.

\section{De controles y terrores}

La conducción de conductas, la circulación por espacios de seguridad y la normalización junto a la 'crisis de las instituciones disciplinarias' son a su vez precondiciones de los cambios que Deleuze enumera para hablar de "nuevas fuerzas que están golpeando la puerta. Son las sociedades de control las que están reemplazando a las sociedades disciplinarias" (Deleuze, 1991: 1).

Estas Sociedades de Control que se corresponden con el neoliberalismo (Rodríguez, 2008) y que operan sobre "máquinas de tercer tipo" (informáticas) cortan la secuencia de la sociedad disciplinaria donde siempre se "estaba empezando de nuevo" en función de unas sociedades de control donde "nunca se termina nada", donde

“(...) la empresa, la formación y el servicio son los estados existentes de una misma modulación (...) los encierros son moldes, módulos distintos, pero los controles son modulaciones, como un molde autodeformante que cambiaría continuamente (...) [y donde] el control es a corto plazo y de rotación rápida, pero también contínuo e ilimitado, mientras que la disciplina era de larga duración, infinita y discontinua" (Deleuze, 1991: 1-3).

En este escenario, la expansión de estas máquinas informáticas que expresan una mutación del capitalismo, permitirán que esta modulación constante se ejerza por fuera de los espacios de encierro, "al aire libre y en todo momento", a todos los "hijos de vecino" en su circulación (Foucault, 2006).

Cabe destacar la importancia del miedo y el terror a la hora de hablar de las Sociedades de Control y vigilancia contemporáneas. Tanto el miedo, la inseguridad y el terror operan como mecanismos de gubernamentalidad que permitan justificar muchas de las tecnologías, regulaciones legales y prácticas implementadas, y asegurar su legitimación. En este sentido se 
puede observar como diversos énfasis sobre el "terror" causado e incentivado por diversos grupos (en un espectro que va desde grupos "terroristas" a delincuentes ordinarios) son la punta de lanza para implementar diversas legislaciones (3), para incentivar la instalación de cámaras de vigilancia y otros dispositivos que permitan monitorear e identificar en todo tiempo y lugar las acciones de individuos y grupos (Alcántara, 2009), que permitan extender y modificar el concepto de "seguridad" al establecer un Estado de Seguridad (Agamben, 2015) donde el estado de excepción se vuelve regla y donde

\begin{abstract}
“(...) la seguridad que está en cuestión hoy no apunta a prevenir los actos de terrorismo sino a establecer una nueva relación con los hombres, que es la de un control generalizado y sin límites (...) Esto significa que al Estado de seguridad le interesa que los ciudadanos cuya protección debe asegurar- permanezcan en la incertidumbre sobre aquello que los amenaza, porque la incertidumbre y el terror van de la mano" (Agamben, 2015: 3).
\end{abstract}

\title{
Psycho Pass: la ventana tecnológica indiscreta
}

"El Sistema Sybil funciona a base del procesamiento en paralelo distribuido por servidores instalados a lo largo del área metropolitana. Un sistema modelo que hace a la tolerancia cero a

fallos una realidad".

Sistema Sybil

Psycho Pass nos transporta a un futuro próximo situado en Japón donde, para asegurar una armonía social que permita terminar definitivamente con la delincuencia, el odio y los asesinatos, se ha construido e instaurado el Sistema Informático Sybil como fuente de paz y de estabilidad para regir las vidas de los ciudadanos.

Cada ciudadano/a es evaluado constantemente gracias a su Psycho Pass, un parámetro biométrico que permite corroborar su estado mental y anímico así como su personalidad y su potencial- predilección para cometer crímenes.

Esta medición es calculada en torno a un número denominado Coeficiente de Criminalidad, que indica si esa persona es un 'buen y correcto ciudadano' -valor menor a 100-; un criminal "en potencia" -valor entre 100 y 300 - o un sujeto peligroso para la sociedad y por tanto eliminable valor mayor de $300-$.

A medida que nos vamos adentrando en los capítulos de esta serie, podemos ver como toda la sociedad japonesa se encuentra estructurada en torno a estos parámetros de criminalidad y por el Sistema Sybil, que lo mide y calcula a través de diversos mecanismos y dispositivos tecnológicos, especialmente en lo que refiere a la vida y el accionar cotidiano de los individuos. 
Cada uno de ellos/as debe medir sus acciones, sentimientos y palabras para no nublar su Psycho Pass y aumentar su valor, tomando todas las precauciones posibles para ello. Así, el anime nos muestra diversas prácticas ejecutadas, desde intentar vivir una vida sin preocupaciones ni cuestionamientos, pasando por aceptar los juicios y exámenes de aptitud tomados por Sybil donde se determina el futuro laboral de cada persona, hasta la práctica del psicoanálisis constante o incluso la toma de medicación 'recomendada' para reducir su nivel de estrés.

Se nos sitúa en una configuración social (Elias, 1989) donde el parámetro de normalidad está establecido y hasta cuantificado por el Psycho Pass de acuerdo al valor central biopolítico del "stress" como parámetro de normalización (Foucault, 2006), medido y calculado por los propios dispositivos tecnológicos del Sistema Sybil. En este sentido, Sybil crea y despliega una red de relaciones de poder en base a su "saber tecnológico-científico" (y por tanto incuestionable) y centrado en su hegemonía del cálculo del estrés para determinar el coeficiente de criminalidad, que organiza todas las acciones de la vida cotidiana (4).

En definitiva, frente a estos parámetros de normalidad/anormalidad determinados ad hoc por el mismo sistema, se despliegan toda una serie de prácticas correctas, deseables y/o posibles para que los individuos puedan intentar 'controlar' este valor y seguir sus vidas 'con normalidad'.

\section{De la tecnología}

"Mediante el Sistema Sibyl, hemos creado un mundo seguro como ningún otro en la historia de la humanidad. Hoy el mundo disfruta de una prosperidad estable, con gran felicidad, para la mayoría de las personas".

Sistema Sybil

La tecnología juega un rol trascendental en el anime, tanto para organizar la vida de cada persona como también para monitorear los cambios en estos parámetros y castigar de acuerdo a la ley cuando estos son excedidos.

Vemos así que cada ciudadano posee una Inteligencia Artificial (IA) personal en forma de un simpático asistente que se encarga de todas las necesidades básicas del hogar: de preparar la comida, de realizar mediciones de sus hábitos y consumos, de recomendarle vestuario o la compra de productos y hasta de ambientar la habitación de forma de hacerla más confortable para el usuario.

Asimismo la compra de vestimenta $u$ otros elementos también parece estar mediado por esta IA y por otros aparatos. En este sentido, la tecnología parece proveer al ser humano de la máxima comodidad y facilidad para evitar todo tipo de estrés cotidiano, entregando a cambio su 
potencial para pensar y actuar en contra de un orden social visto quizás como algo no tan justo y perfecto.

Fuera del hogar, los automóviles se conducen solos, cámaras de videovigilancia monitorean constantemente el Psycho Pass de los individuos e incluso drones y robots policíacos revisan constantemente los diversos espacios, de forma de asegurarse que no haya ningún aumento del coeficiente de criminalidad de individuos o grupos. Para ello, cuando son obligados a actuar asumen la forma de amables y simpáticos avatares para hacer lo más amena posible una situación de conflicto y estrés.

Cabe destacar la existencia de plataformas de redes sociales, pero con una modalidad y lógica distinta a las que existen en nuestra sociedad. Por el lado de la modalidad, el ingreso a las mismas se realiza con un casco de inmersión total, similar a los utilizados por los juegos de realidad virtual, donde el usuario crea un avatar y tiene la posibilidad de transitar diversos canales personalizados de otros usuarios. Asimismo la lógica que parece mantenerse es la del anonimato, en sentido de utilizar un avatar con una figura de fantasía que impida detectar fácilmente a su usuario real; lógica mantenida en los encuentros masivos cara a cara donde cada asistente aparece vestido de su avatar. En este sentido, las redes sociales que muestra el anime tienden a parecerse más a los viejos canales de chats/IRC donde el usuario puede entrar de forma anónima a diversos canales, mantener conversaciones privadas, etcétera. Sin embargo se agrega a eso algo de la lógica actual teniendo la posibilidad los usuarios más "famosos" de cobrar donaciones de parte de sus seguidores, de forma similar pero a la vez diferente al modelo profesional de los youtubers actual.

Podemos analizar cómo los diversos dispositivos y posibilidades tecnológicas obran como extremidades de Sybil para mantener el control y la vigilancia de los sujetos "al aire libre y de forma constante" (Deleuze, 1991) pero también en la intimidad del hogar, diluyendo la separación público-privado. Los individuos son libres de circular y de ejecutar las acciones correctas y deseables (dejarse asistir por las IA personalizadas, no conducir sus automóviles, pasear "sin preocupaciones", etcétera) o posibles/permitidas (ingresar a las redes sociales), siempre teniendo en cuenta que en todo momento y lugar sus acciones y estrategias deben ser auto-medidas para no aumentar su coeficiente criminal y por tanto, para evitar traer sospechas de un "mal accionar" o para evitar convertirse directamente en criminales potenciales pasando la línea de la "normalidad" establecida. Es así como la tecnología se convierte en herramienta y monitor a la vez de todas las acciones, sentimientos y pensamientos de los ciudadanos.

\section{Agencia de Seguridad Pública, dominators y los perros de caza}


"Todos ustedes funcionan como una terminal más del sistema. Son la parte que interactúa con la sociedad. Así es como lo reconocen y lo entienden. Si alguno de ustedes dudara del Dominator, eso podría provocar que todos los ciudadanos dudaran del sistema". Joshu Kasei, supervisora de inspectores

Entrando en la trama central del anime, en todo orden por perfecto que se enuncie hay riesgos de violaciones al mismo, y para eso existe la figura de la Agencia de Seguridad Pública, un organismo del gobierno que ante una alarma de un coeficiente criminal alto 0 ante una situación que pueda perturbar a otros coeficientes "normales", envía a sus empleados humanos- a investigar y solucionar el problema.

Entre estos podemos encontrar a los inspectores, ciudadanos con capacidades deductivas y morales supuestamente notables a los que el Sistema Sybil les dio la posibilidad de acceder a ese trabajo (entre otros o solo ese) encontrándolos "aptos e idóneos" para el mismo. Ellos comandan y dirigen a los ejecutores, criminales en potencia previamente detectados y aislados, a quienes se les otorga una oportunidad de "contribuir con el orden social" atrapando o eliminando a otros criminales.

La herramienta para llevar su labor es un pistola tecnológica denominada Dominator conocida como "los ojos de Sybil", ya que mediante este aparato el usuario puede medir directamente el coeficiente de criminalidad de la persona a la que apunta, siendo el arma la que determina si es un objetivo válido o no, si debe entrar en modo paralizer (ante criminales potenciales con valor 100 a 300), en modo eliminator ante criminales irrecuperables (300 o más de coeficiente de criminalidad) u objetos tecnológicos peligrosos (modo decomposer).

Ante una situación problemática son los ejecutores los que deben actuar, siendo los inspectores meros supervisores de dicho accionar, en parte porque se da por sentado que los ejecutores al tener su mente "contaminada" pueden comprender e interpretar mejor las lógicas de los criminales en potencia o irrecuperables a atrapar y también como medio de evitar que se nuble y aumente el coeficiente de criminalidad de los inspectores. Estos a su vez pueden disparar para incapacitar a un ejecutor si consideran que estos realizaban algún acto inapropiado.

La serie nos brinda una gran cantidad de casos policíacos a resolver por la División I de la Agencia de Seguridad Pública, donde diversos ciudadanos con un coeficiente de criminalidad elevado realizan acciones contra la ley o que puedan afectar el coeficiente de otros. En este sentido la regla es clara: victimario pero también víctima deben ser juzgados por el dominator. no importa que uno no haya hecho nada malo, si su coeficiente de criminalidad excede el valor de la normalidad uno es un potencial criminal. Es decir, aunque acabe de ser víctima de violencia física si el coeficiente de criminalidad aumenta uno es el culpable por no poder mantener su Psycho Pass estable y sereno y por tanto debe ser castigado. 
Al igual que evidencia la frase con la que se abre este subapartado, los inspectores y los ejecutores son las extremidades humanas del Sistema Sybil que complementan a las extremidades tecnológicas mencionadas en el subapartado anterior.

En este sentido, el dominator como "ojos de Sybil" obra de juez y ejecutor en el mismo momento y lugar, en base al coeficiente de criminalidad (parámetro de normalización) que el mismo dispositivo juzga. Sin embargo, el disparo no es automático sino que debe ser operado por un inspector o ejecutor, lo que también brinda un pequeño margen de posibilidad de acción de estos sujetos, aunque la premisa inicial parece ser que si el dominator indica tal o cual cosa esa es la realidad y por tanto es lo que debe hacerse, obturando que esta resolución es a base de la construcción de un cálculo de acuerdo a diversos parámetros de saber, poder y verdad ejercidos por el Sistema Sybil. De ahí la eficacia de la construcción y ejercicio de poder.

Es interesante pensar en la interdependencia del poder (Elias, 1990) en esta red de configuraciones y relaciones, ya que Sybil opta por utilizar a varios 'criminales en potencia' ya demostrados como forma de intentar mostrar que todos tienen una función y como forma de mecanismo de preservación de los inspectores. Esto es parte de un aprendizaje de la misma experiencia y configuración de Sybil, ya que en el anime se relata que en los inicios de su implementación, la mayoría de los inspectores (y sus psycho pass) quedaban fuertemente afectados por el cumplimiento de su rol. Como respuesta a esto, fue posteriormente creada e incorporada al sistema la figura del ejecutor como solución a este problema.

Cabe teorizar que Sybil necesita que exista la figura del "criminal en potencia" ya que el miedo/terror que genera tanto su mera figura como la posibilidad potencial de que cada ciudadano pueda devenir en uno, es la argumentación que hizo que el sistema fuera creado y que mantiene la legitimidad de su accionar y supervisión social, instalando un Estado de Seguridad (Agamben, 2005).

Por ende, no solo vemos una interdependencia entre Sybil y estos "criminales en potencia" (con sus lógicos equilibrios de poder a favor del sistema) sino también una interdependencia entre Sybil y sus extremidades tecnológicas y humanas, siendo estas últimas las que complementan el "argumento tecnológico" de la legitimidad del sistema.

\section{Del poder, la resistencia y el alma humana}

"Al analizar el campo de fuerza de un organismo vivo leído por un escáner cimático, dedujeron cómo funciona la mente de una persona. La ciencia finalmente descubrió el secreto detrás del alma humana, y como resultado esta sociedad cambió drásticamente. Sin embargo, la voluntad de las personas no forma parte de esa valoración".

Makishima Shogo 
Así como "todo poder engendra su resistencia" (Foucault, 2002) podemos agregar que "en todo rebaño de ovejas siempre existe la oveja negra". En este anime, esta figura es Makishima Shogo, un intrigante personaje que, a fuerza de diversos planes y acciones impulsadas por planteos filosóficos y otras motivaciones, busca "revelar el secreto del alma humana" en una sociedad que se ha entregado a la mera existencia conducida por la tecnología y los parámetros fijados por el Sistema Sybil.

Makishima es una producción de la configuración de relaciones sociales y de poder del Sistema Sybil ya que a pesar de sus pensamientos, discursos y actos contra el orden establecido su Psycho Pass no se nubla ni asciende, haciéndolo invulnerable a los dominators y representando una falla en el discurso de "perfección". Esta circunstancia, denominada en la serie como "criminal asintomático" no sólo generó el impulso inicial de Makishima por poner en jaque el sistema, sino también es enunciado como un secreto que debe ser mantenido oculto a los ojos del público, so peligro de que tambalee todo el sistema establecido en torno a la premisa de la medición del coeficiente de criminalidad.

De esta forma, nuestros protagonistas de la División I irán sorteando diversos casos donde se pondrán en juego las vidas humanas y el cuestionamiento por el orden social. Makishima obra en gran parte de la serie como maestro estratega por detrás de dichas situaciones, siendo quien simplemente da armas, lugares o recursos a aquellos que ya tienen un motivo también producido directa $\mathrm{o}$ indirectamente por la red de configuraciones sociales y de poder desplegada y ejercida por Sybil.

Este "estar por detrás" se hace para evidenciar que él simplemente facilita el poder expresar todos esos sentimientos reprimidos producidos por este "perfecto sistema", a la vez que le sirve en su investigación y análisis de "lo que el ser humano puede llegar a hacer". Sin embargo, en otras situaciones toma un papel central ya que considera que "es aburrido un juego en el que no puedes jugar".

\section{La disrupción como forma de resistencia. Del caos y la desinformación}

La primera situación a analizar se hace presente en el capítulo 14, cuando se ve a un hombre con un casco primero ingresar violentamente a una farmacia y asesinar a su personal sin que ninguna alarma sea disparada ante una suba de Psycho Pass en la zona. Se nos revela así que este casco obra de dispositivo que anula la medición del coeficiente de criminalidad. Más tarde, el mismo sujeto camina por la calle, golpea a una mujer con un martillo frente a una multitud de personas que circulan y se quedan atónitos sin comprender la situación. El hombre del casco golpea furiosa y repetidamente a la mujer sin reacción alguna de los concurrentes. Uno de los drones policíacos cercanos se acerca, pero en lugar de detener al agresor, activa 
su simpático avatar y le indica a la mujer que su coeficiente de criminalidad estaba subiendo y le sugiere tomar tratamiento terapéutico, ignorando al hombre del casco y su salvaje accionar, mientras se muestra que los martillazos acaban con la vida de la joven. Asimismo, un cómplice de Makishima graba con su teléfono la escena y rápidamente sube el video a las redes. El capítulo 15 arranca con el video siendo viralizado y fruto de múltiples comentarios confusos de parte de los ciudadanos, que no pueden procesar los eventos que van surgiendo.

Tras esto, se nos muestra como Makishima distribuye a varios grupos de personas los cascos anti-lectura del Psycho Pass, iniciando estos grupos una serie de ataques, robos, saqueos, asesinatos, etcétera, expandiendo el caos por toda la ciudad. Estos hechos nuevamente se vuelven virales en las distintas redes sociales, donde se ve como los ciudadanos confundidos comentan la situación, preguntando si la Agencia de Seguridad Pública está tomando cartas en el asunto, o incluso qué elemento casero puede servir como arma de defensa logrando insólitas respuestas (ejemplo: una planta). Tras diversos casos, se nos muestra a grupos de ciudadanos enfrentando a los sujetos con casco pero incluso también asesinando a ciudadanos sin este elemento a quienes creen haber visto "quitándose el casco". Tras esta situación y la duda existencial de si eso no les generará futuros problemas de aumento de su coeficiente criminal, uno de ellos menciona que "leí en la red que auto-defenderse o asesinar criminales disminuye el coeficiente criminal" con lo cual da a entender no sólo que la auto-defensa es posible sino hasta deseable/correcta, ya que compatibiliza con la estructuración del mundo en torno al coeficiente de criminalidad del Psycho Pass. A su vez en esta situación el anime nos da una pequeña muestra de lo que la desinformación en las comunicaciones en internet pueden hacer.

Tras la organización de una estrategia y planificación para reprimir los eventos de caos se nos muestra una acción con éxito de parte de la División I, tras la cual el ejecutor Kogami menciona que tanto ciudadanos comunes como los sujetos con casco son víctimas, ya que estos elementos fueron el resorte que impulsó a estos últimos a actuar y a dar rienda suelta a realizar acciones de "venganza" ante la "injusticia" del sistema Sybil, situación que no se hubiera dado sin esta oportunidad.

\section{Desnudando al rey}

La trama que sigue a la situación anterior se despliega mayormente en los capítulos 16 y 17 donde se evidencia que el caos fue una estrategia para dejar desprotegida la Nano Tower, donde opera el Ministerio de Bienestar a cargo del Sistema Sibyl. Allí ingresan Makishima y sus colaboradores, uno de los cuales es un cracker informático con altos conocimientos en la materia y con la teoría de que Sybil no es un sistema descentralizado sino justamente que tendría su núcleo en dicha torre. Tras diversas situaciones y enfrentamientos, el secuaz de 
Makishima logra al fin revelar la verdadera forma del Sistema Sybil: una serie de cerebros humanos interconectados intercambiados constantemente que se comunican entre sí. Es evidente la emoción del secuaz de Makishima al grabar la escena con su teléfono móvil, haciendo alusión a que cuando esto sea revelado se caerá el argumento "tecnológico" que da sustento al orden social encarnado y dirigido por Sybil. A su vez la narración nos revela a los espectadores que la directora Kasei de la Agencia de Seguridad Pública es un cíborg (posteriormente se nos informa que este cuerpo contiene uno de los cerebros que conforman Sybil, siendo una práctica común que se vayan intercambiando los cerebros de acuerdo a la situación) y que puede hacer que el dominator ignore el coeficiente de criminalidad para entrar en modo eliminator, el cual utiliza para eliminar a los únicos dos testigos de la escena. Así como Sybil hace las reglas, también crea sus excepciones.

Tras otra serie de hechos paralelos que concluyen con la captura de Makishima, se nos muestra luego un diálogo entre Makishima y la directora Kasei (portando un cerebro de un antiguo colaborador) donde le explica que los cerebros que componen al Sistema Sybil son los de todos aquellos criminales asintomáticos que han sido capturados a lo largo del tiempo, dado que estos son "sujetos con una personalidad irregular que no encaja con los otros seres humanos, son capaces de ver las acciones de otros como observadores y árbitros", revelando que el Sistema Sybil se produce y reproduce a sí mismo mediante esta operación.

En este sentido, la narración no solo nos termina de desarmar el "argumento tecnológico" que opera de base de la configuración social operada por Sybil, sino que le agrega que son los criminales "más peligrosos" (por no ser cuantificable su coeficiente de criminalidad) aquellos que componen el sistema que crea y mantiene el orden.

Para cerrar la escena, cabe destacar la negativa de Makishima y su posterior escape antes, destruyendo el cerebro de su antiguo colaborador.

\section{Concluyendo, reflexionando e invitando}

"Vaya mundo en el que vivimos, ¿Verdad?, pero podría ser peor, ¿Verdad? Así es -dije-, o peor aún, podría ser perfecto". William Gibson, The Gernsback Continuum

Para concluir este artículo, no nos adentraremos en el enfrentamiento final entre Makishima y la División I sino que procederemos a redondear el análisis de este anime de ciencia ficción.

Por un lado, cabe destacar que la configuración de relaciones sociales y de poder ordenada y ejercida por el Sistema Sybil no surge de la nada, sino que representa un estado de "paz" (Marin, 1996) luego de diversos conflictos que han derivado en la victoria de ciertos sectores 
que han impuesto sus reglas de juego como parámetros de normalización, saber y verdad y de lo que es correcto/deseable, posible e imposible al resto de los individuos. Así, esta "paz" no solo es inestable sino que incluso genera las propias armas materiales y morales que permiten reproducirla pero a su vez combatirla. En este sentido, vemos cómo la tecnología (los cascos anti-lectura del Psycho Pass, las comunicaciones online y la desinformación en ellas, entre otras) sirve y obra como recursos y estrategias de resistencia para encontrar diversos "puntos ciegos" en la configuración del orden, que permitan develar la gubernamentalidad de conductas establecidas en torno al parámetro del Pyscho Pass y de la lectura de su coeficiente y de ese modo desnivelar las balanzas de poder de esta "paz" establecidas por el Sistema Sybil.

Asimismo, la narrativa del anime constantemente nos muestra los efectos -previstos e imprevistos- de poder de la producción de sujetos, cuerpos y conductas. Para los ciudadanos "comunes", a quienes se los muestra constantemente despreocupados y confiados en la efectividad del accionar tecnológico ( $y$ humano) de Sybil y sus extremidades para mantener el orden, parecería que cualquier situación que esté por fuera de los parámetros que ordenan la sociedad les son sumamente difíciles de comprender, al no tener una disposición para la acción correcta/deseable o al menos posible para su accionar en respuesta de dicha situación. Por eso muchas veces vemos como son meros espectadores atónitos, víctimas o peones de los principales actores en juego del anime. Para los efectivos de la Agencia de Seguridad Pública, vemos claramente la diferencia entre los ejecutores "contaminados· y los inspectores "puros" que batallan por mantener el orden e intentar mantener su convicción de ello.

Para los antagonistas podemos hablar de dos categorías. Por un lado Makishima y otros criminales asintomáticos, fiel evidencia de la producción de Sybil, son quienes canalizan como forma de boicot al orden su descontento por no encontrar su "papel en la sociedad" debido a la ineficaz lectura de su Psycho Pass. Pero también el anime nos muestra a otros antagonistas menores (cuyo coeficiente sí puede ser medido) quienes realizan diversos actos en venganza de otras personas o del mismo sistema, ya que directa o indirectamente sus personas y trayectorias de vida se han visto perjudicadas por la forma de configuración social dominante. En este sentido, es que vemos a la figura de Makishima como director de orquesta, proporcionando las armas materiales y morales para que estos sujetos puedan llevar a cabo su accionar, el cual parecería que sin su intervención no tendría lugar o no tendría la misma efectividad y visualización.

Se puede evidenciar que en todo el artículo no se usó la palabra/categoría distopía -sociedad futura controlada y restrictiva y por tanto indeseable-, para referir a Psycho Pass, ya que desde otro punto de vista podría considerarse una utopía -sociedad futura deseable que vale la pena buscar-. Sin intención de que se considere demente al autor de estas líneas, simplemente podemos postular que la distopía de unos es la utopía de otros, ya que la sociedad regida por Sybil expresa muchos de los pedidos mayoritarios actuales: paz, seguridad, orden, previsión y 
estabilidad. Saliendo del anime, estos pedidos y parámetros son los que en parte permiten la instalación del Estado de Seguridad (Agamben, 2015) donde la "excepción se ha vuelto regla"; estados constituidos y reivindicados como democráticos.

Asimismo, muchos de los avances y dispositivos tecnológicos de hoy día como los teléfonos móviles, las redes sociales, las impresoras 3D, las cámaras de vigilancia callejeras, los drones, los electrodomésticos con tecnología de Internet de las cosas, los dispositivos biométricos entre un gran etcétera no están muy lejos -al contrario, lo hacen (Gendler, 2015)- de permitir cuantificar y determinar nuestros perfiles como deseables, posibles, incorrectos y potencialmente eliminables sin necesidad de un dominator o de un psycho pass.

La ciencia ficción en general nos permite ver muchos de los miedos y anhelos de nuestra sociedad actual, dando como resultado que Psycho Pass sea una posible ventana a un futuro no tan lejano.

El análisis en profundidad de un anime como narración audiovisual de ciencia ficción nos permite develar y comprender muchos puntos en común presentes en nuestras sociedades contemporáneas y nos abre una ventana a poder visualizar uno de los futuros posibles en gestación.

Se invita al lector y a los/as diversos/as colegas a embarcarse en esta aventura o en otras del género, a riesgo de que aumente su coeficiente de criminalidad, claro está, en pos de la batalla por las almas y la moral de determinar que es una vida que merezca ser vivida.

\section{Notas}

(1) Salvo notables (y parciales) excepciones como Jean Baudrillard.

(2) Cabe aclarar que se trabajará solo con la primera temporada de esta obra sin considerar la segunda temporada y la película.

(3) Como la Ley Patriótica de los Estados Unidos en 2006, la cual brinda poderes extraordinarios al Estado y a sus diversas agencias de seguridad para la detención y procesamiento de individuos y colectivos sin juicio previo.

(4) Un ejemplo de esto es que los hogares ya no poseen cerradura, no existen detectores de armas, etcétera, dado que se presupone que un ciudadano con un coeficiente de criminalidad elevado será capturado o eliminado de forma rápida y eficiente antes de poder cometer un crimen.

\section{Bibliografía}

Agamben, G. (23 de diciembre de 2015). Del Etat de droit à I Etat de sécurité. Le Monde. Recuperado de http://artilleriainmanente.blogspot.com.ar/2015/12/giorgio-agambendelestado-de-derecho.html 
Alcántara, J. (2009). La Sociedad de Control: privacidad, propiedad intelectual y el futuro de la libertad. Barcelona: El Cobre ediciones.

Asimov, I. (1986). Sobre la Ciencia Ficción. Barcelona: EDHASA.

Bourdieu, P; Chamboredon, J-C y Passeron, J-C. (2002). El oficio de sociólogo: presupuestos epistemológicos. Buenos Aires: Siglo XXI.

Deleuze, G. (1991). Posdata a las sociedades de control. Recuperado de http://www.fundacion.uocra.org/documentos/recursos/articulos/Posdata-sobre-lassociedades-de-control.pdf

Elias, N. (1989). El proceso de la civilización. Investigaciones sociogenéticas y psicogenéticas. México: FCE.

Elias, N. (1990). La sociedad de los individuos. Barcelona: Península.

Elias, N. (1994). Conocimiento y poder. Madrid: La Piquéta.

Foucault, M. (1980). Du gouvernement des vivants. En Dits et Ecrits 1954-1988 (Tomo IV, pp. 125-129). Paris: Gallimard.

Foucault, M. (1981). Un diálogo sobre el poder y otras conversaciones (introducción y trad. Miguel Morey). Madrid: Alianza Editorial.

Foucault, M. (1984). Como se ejerce el Poder. En Dreyfus, H; Rabinow, P y Foucault, M. Un Parcours Philosophique, Paris: Editions Gallimard. Recuperado de http://www.unizar.es/deproyecto/programas/docusocjur/FoucaultPoder.pdf

Foucault, M. (1987). Historia de la sexualidad. 1. La voluntad de saber. Madrid: Siglo XXI.

Foucault, M. (1996). Genealogía del racismo. La Plata: Altamira.

Foucault, M. (2002). Vigilar y Castigar. Buenos Aires: Siglo XXI Editores.

Foucault, M. (2006). Seguridad, territorio, población. (Curso en el Collège de France). Buenos Aires: Fondo de Cultura Económica.

Gendler, M. (2015). ¿Qué es la Neutralidad de la Red? Peligros y potencialidades. Revista Hipertextos, 2(4), pp. 137-167.

Gibson, W. (1981). The Gernsback Continuum. En Carr, T (editor). Universe 11. Nueva York: Doubleday.

Marín, J.C. (1996). Conversaciones sobre el poder: una experiencia colectiva. (Volumen I). Instituto de Investigaciones Gino Germani, Universidad de Buenos Aires: Buenos Aires.

Rodríguez, P. (2008). ¿Qué son las sociedades de control? Revista Sociedad, 27, Buenos Aires, Prometeo/Facultad de Ciencias Sociales (UBA). Recuperado de http://www.sociales.uba.ar/wp-content/uploads/21.-Qu\%C3\%A9son-las-sociedades-decontrol.pdf

Sibilia, P. (2005). Biopoder. En El hombre postorgánico. Cuerpo, subjetividad y tecnologías digitales. Buenos Aires: Editorial Fondo de Cultura Económica.

Yamamoto, K. (productor) (2012). Psycho-Pass. Tokio, Japón. 